\title{
Banks performance evaluation: A hybrid DEA-SVM- The case of U.S. agricultural banks
}

\author{
Kekoura Sakouvogui ${ }^{\mathbf{a}^{*}}$
}

${ }^{a}$ North Dakota State University, United States

\begin{tabular}{l}
\hline C H R O N I C L E \\
\hline Article history: \\
Received August 3, 2018 \\
Received in revised format \\
August 112018 \\
Accepted September 72018 \\
Available online \\
September 72018 \\
\hline Keywords: \\
Data envelopment analysis \\
DEA \\
Efficiency \\
Bank \\
SVM
\end{tabular}

\section{Introduction}

In economic theory, the efficient and effective utilization of resources are the main objectives of every bank. The study of bank efficiency has shown to be important during the recent financial crisis of 20072009, which not only impacted the United States (U.S) but also Europe and the whole world. Since then, the prediction of bank failures has become an important issue studied by researchers (Ataullah \& Le, 2006). Previous studies have produced mixed results regarding the effects of efficiency in the banking sector (Drake, 2001; Hao et al., 2001; Ataullah and Le, 2006; Andrews \& Pregibon, 1978). Hence, frontier efficiency analyses have become preferred methods of evaluating performance in the banking sector. Efficiency benchmarking allows banks to estimate production, cost and profit functions. There are two main techniques used to evaluate these efficiencies: parametric methods, exemplified by the Stochastic Frontier Analysis (SFA), and the non-parametric methods exemplified by Data Envelopment Analysis (DEA). DEA, a non-parametric method based on the linear programming framework, can manage complex production environments with multiple inputs and outputs. On the other hand, SFA is a statistical method that can discriminate between efficient units,

\footnotetext{
* Corresponding author.

E-mail address: kekoura.sakouvogui@ndsu.edu (K. Sakouvogui) 
and decomposes the statistical error, $\varepsilon$, into a noise term, $v$, and an inefficiency term, $u$. DEA has an advantage over SFA because it does not account for a statistical error term. Hence, it does not deal with the distributional assumptions of $u$ and $v$. This paper is concerned with the DEA approach.

A fundamental assumption of the DEA method is that the decision-making units (DMUs) such as banks in a sample must all have a functional similarity. However, this can become problematic in the presence of noise (Fried et al., 2002). Moreover, given the amount of data available, literature has shown that there is still a need to address the importance of noise on the performance of DEA measures. ${ }^{1}$ Proponents of DEA have suggested integrating machine learning techniques with DEA efficiency measures to alleviate the issues of noise (Wu et al., 2006; Azadeh et al., 2007; Favero \& Papi, 1995). One such technique that has shown good performance in the prediction/classification of the financial markets is Support Vector Machine (SVM) (Cao and Tay, 2003; Racine, 2000). SVM has a literature that is relatively small compared to other statistical methods such as Random Forest, K-Nearest Neighbor, and neural networks (Boyacioglu et al., 2009). The recent approach of integrating DEA efficiency measures with SVM has some drawbacks including uncontrolled dependence of the efficiency measures. Hence, in this paper, a new combination of DEA and SVM method with four kernel functions (linear, sigmoid, polynomial and Radial Basis Function (RBF)) is proposed. Our research contributes to the literature by accounting for the heterogeneity effect in the efficiency measures while applying SVM methodology to assess any inconsistency between the efficiency estimates produced using the U.S. Federal Agricultural Banks data from 2005 to 2016.

In this paper, our contribution is in three-fold: First, we estimate the efficiency measures. Second, to account for heterogeneity among the banks, we determine the optimal number of cluster using the gap statistical method and then cluster the efficiency measures of the U.S Federal Agricultural Banks prior to the financial crisis (2005-2006), during the financial crisis (2007-2009) and post the financial crisis (2010-2016) using k-means algorithm. Third, we integrate the DEA efficiency measures estimated with SVM while accounting for four variety of kernel functions: linear, sigmoid, polynomial, and Radial Basis Function (RBF). The remainder of this paper is structured as follows: Section 2 introduces the DEA and SVM model, and the integration of DEA and SVM. Section 3 presents the empirical data set and the input and output variables. Section 4 presents the results. Section 5 summarizes the research and provides additional discussion.

\section{Theoretical framework}

Primal production theory assumes that the relationship between multiple outputs, $y=\left(y_{1}, y_{2}, \ldots, y_{j}\right) \in \mathfrak{R}_{+}^{J}$ and inputs, $x=\left(x_{1}, x_{2}, \ldots, x_{i}\right) \in \mathfrak{R}_{+}^{I}$ is reflected by the concept of production function. The production function framework forms the bases in the estimation of the DMUs efficiency using linear programming DEA.

\subsection{DEA model}

The technology that transforms inputs into outputs can be represented by input set $L(\mathbf{y})$. The input set satisfying constant returns to scale and strong disposability of input is defined as:

$$
L(\mathbf{y})=\left\{\mathbf{x}: \mathbf{y} \text { is produced by } \mathbf{x} ; \quad \mathbf{x} \in \mathfrak{R}_{+}^{I} \quad \mathbf{y} \in \mathfrak{R}_{+}^{J}\right\}
$$


The input set $L(\mathbf{y})$ denotes the collection of input vector that yield output vector. This concept is represented by an input distance function evaluated for any DMU a reference production possibility set $T$, as:

$$
D_{i}^{T}\left(y^{t}, x^{t}\right)^{-1}=\min \left\{\lambda: \lambda x^{t} \in L^{T}\left(y^{t}\right)\right\}
$$

or

$$
\min _{\theta, z} \text { st. } \quad y^{t} \leq Y z \quad Y=y_{1}, \ldots, y_{T}
$$

subject to

$$
\begin{aligned}
& \lambda x^{t} \geq X z \quad X=x_{1}, \ldots, x_{T} \\
& z \geq 0
\end{aligned}
$$

Here, the second expression of Eq. (2) identifies the linear program that is used to calculate the distance function, with the $z$ 's being a $T x l$ vector of intensity variables that identify the constant return to scale (CRS) boundaries of the reference set. Once the traditional DEA analysis has been performed it may be difficult to interpret the efficiency measures obtained for each bank because of the non-homogeneity of the banks. In our paper, we solve the issue of heterogeneity by determining the optimal number of clustering group within the years using the gap statistic method, first by developed by Tibshirani, et al., (2001). The results suggest that the efficiency measures can be classified into four groups: Highly Efficient (HE), Efficient (E), Highly Inefficient (HI), and Inefficient (I).

\subsection{Support Vector Machine}

Support Vector Machine (SVM), a relatively young classification algorithm that has been proposed by Vapnick (Xu et al., 2006), is devised to provide a computationally efficient way of separating hyperplanes in a high dimensional feature space. Given a training data $\left(X_{1}, y_{1}\right) \ldots .,\left(X_{n}, y_{n}\right)$ where $X_{i} \in \mathrm{R}^{m}$ and $y_{i} \in \mathrm{R}$, the goal is to find a function to classify $\mathrm{g}(\mathrm{x})$ where:

$$
g(x)=w^{T} \Phi(X)+b
$$

where $\varphi(i): \mathrm{R}^{m} \rightarrow \mathrm{R}$ and $w$ and $b$ are the parameters learned from the training data. $w$ is the weight that defines a direction that is perpendicular to the hyperplane, $b$ is the bias term that moves the hyperplane parallel to itself and $x$ is the support of the support machine. In the binary classification ${ }^{2}$ with $y_{i} \in$ $\{-1,+1\}$ corresponding to the class label of $x_{i}$, the function margin that is defined as the margin measured by the function output of $g(x)$ is:

$$
\mathrm{g}(\mathrm{x})=\left\{\begin{array}{l}
\left\langle w \cdot x^{+}+b\right\rangle=+1 \\
\left\langle w \cdot x^{-}+b\right\rangle=-1
\end{array}\right.
$$

The goal of the algorithm is to maximize the distance between the training data that are closest to the decision boundary. The margin of separation is related to the so called Vapnik-Chervonenkis dimension, which measures how complex the learning machine is (Vapnick, 1998). Given a linearly separable training data, the hyperplane $(w, b)$ that solves the optimization problem

$$
\begin{aligned}
& \min _{w, b}\{\langle w \cdot w\rangle\} \\
& \text { subject to } \\
& y_{i}\left[\left\langle w \cdot x_{i}\right\rangle+b\right] \geq 1, \vee i
\end{aligned}
$$

2 In the multi-classification, SVM performs one versus the other classification framework. Therefore, we will always get back to the binary classification framework. 
realizes the maximal margin hyper-plane with a geometric margin $\frac{1}{\|w\|_{2}}$ which is the minimal distance between two classes. The transformation of the optimization problem in (4) into a dual problem gives us the primal Lagrangian:

$$
L(w, b, \alpha)=\frac{1}{2}\langle w \cdot w\rangle-\sum_{i=1}^{l} \alpha_{i}\left[y_{i}\left[\left\langle w \cdot x_{i}\right\rangle+b\right]-1\right]
$$

This dual is found by differentiation with respect to $w$ and $b$, and it is only dependable on the Lagrange multipliers $\alpha_{i}$. Furthermore, Cortes and Vapnick (1995) suggested a modification to the original optimization statement that will penalize the failure of a training data point to reach the correct margin. The proposed modification is conducted by introducing the slack variable that accounts for any data that were wrongly misclassified. As a result, the algorithm could be generalized to a nonlinear classification by the introduction of a kernel function $K$ that maps the input data into a high-dimensional feature space (Vapnik, 1982). The kernels function used in this paper are:

Linear : $K(x, y)=x^{T} y$; Gaussian (RBF): $K(x, y)=\exp \left(-\gamma k x-y k^{2}\right)$; Sigmoid: $K(x, y)=\tanh \left(a+\gamma x^{T} y\right)$; Polynomial: $K(x, y)=\left(\gamma+x^{T} y\right)^{d}$ where $a, \gamma, d$ are the parameters associated with each kernel function.

The empirical framework is as follow:

1. Partition the data into three groups: prior to the financial crisis (2005-2006), during the financial crisis (2007-2009), and post financial crisis (2010-2016).

2. Estimate the efficiency measures assuming an input oriented DEA model by year.

3. Test for stationarity of the efficiency measures across each group of the financial crisis.

4. Using the efficiency measures of each group, a cluster analysis (kmeans) is implemented with four clustering groups

5. Apply SVM classification technique by splitting the data into two sets: training set and a testing set.

6. Using the training set within each group of the financial crisis, perform a grid search to optimize the parameters associated with the kernels of SVM.

7. Apply the trained SVM model to the testing data and calculate the prediction error and the accuracy under the four kernels.

\section{Data and construction of the variables}

This study uses the annual data for most of the agricultural banks located in the U.S from 2005 to 2016. The data was provided by the Farm Credit Administration (FCA) Web Site. Farm Credit System institutions submit Call Reports to FCA on a quarterly basis. These reports contain the institutions financial data. A random sample of 363 banks were selected from 2010-2016, 121 banks were selected from 2005-2006, and 182 banks were selected from 2007-2009.

Within DEA methodology, the efficiency measures are only relative to the best DMUs in the data; that is the choice of the input-output variables (Martić and Savić, 2001). Following the works of Sealey and Lindley (1977), and Casu and Molyneux (2003), we considered the intermediate approach with two inputs: (total interest expenses and total non-interest expenses), and two outputs (total loan and other earning assets). Moreover, the input total cost is measured as the sum of the two inputs variables: total interest expenses and total non-interest expenses. The output, total loan, is measured as the sum of all loan accounts by the banks listed in FCA and the output, other earning assets is measured as the sum 
of total securities (treasury bills, government bonds and other securities), deposits with banks, and equity investments.

\section{Results and Discussions}

\subsection{Unit Roots Test}

SVM is a method that assumes that the data is stationary. In the literature of the unit root tests, the augmented Dickey Fuller (ADF) test of Said and Dickey (1984) and the KPSS test of Kwiatkowski et al., (1992) are the most popular. However, because of the drawback of ADF that is, the ADF test has low power, KPSS test of stationarity is considered in this paper. Hence, the hypothesis for this test can be written as:

Hypothesis 1 each time series follow a straight line time trend with stationary errors.

Hypothesis 2 each time series is non-stationary.

Table 1 shows the results of the KPSS test. While applying the KPSS test, the null hypothesis is not statistically rejected at $1 \%$ for each of the period of the financial crisis. Therefore, we conclude that the efficiency measures are stationary. Hence, SVM can be applied on the efficiency measures obtained during the periods of 2005-2006, 2007-2009, and 2010- 2016.

Table 1

KPSS Test of the efficiency measures for prior, during and post the financial crisis

\begin{tabular}{llll}
\hline & $2005-2006$ & $2007-2009$ & $2010-2016$ \\
\hline p-value & 0.0216 & 0.1 & 0.0172 \\
\hline
\end{tabular}

4.2. Prior to the financial crisis

The efficiency measures of agricultural financial banks for the period of 2005-2006 are evaluated using the input oriented BCC model. Tables 2 summarizes the efficiency measures of banks in our sample by year. Tables 2 shows a significant dynamic change.

Table 2

Efficiency Measure Prior (2005-2006)

\begin{tabular}{|c|c|c|c|c|c|c|c|}
\hline Name of the Bank & Year & $\begin{array}{c}\text { Efficiency } \\
\text { Scores }\end{array}$ & $\begin{array}{l}\text { Cluster } \\
\text { group }\end{array}$ & Name of the Bank & Year & $\begin{array}{c}\text { Efficiency } \\
\text { Scores }\end{array}$ & Cluster group \\
\hline FCB of Texas & 2005 & 1.000 & 4 & Legacy Ag Credit, ACA & 2005 & 0.627 & 3 \\
\hline FCB of Texas & 2006 & 0.951 & 4 & Legacy Ag Credit, ACA & 2006 & 0.804 & 2 \\
\hline AgFirst FCB & 2005 & 0.677 & 1 & First South ACA & 2005 & 0.665 & 1 \\
\hline AgFirst FCB & 2006 & 1.000 & 4 & First South ACA & 2006 & 0.822 & 2 \\
\hline AgriBank, FCB & 2005 & 0.913 & 4 & Central Kentucky ACA & 2005 & 0.719 & 1 \\
\hline AgriBank, FCB & 2006 & 1.000 & 4 & Central Kentucky ACA & 2006 & 0.936 & 4 \\
\hline U.S. AgBank, FCB & 2005 & 0.699 & 1 & Valley ACA & 2005 & 0.803 & 2 \\
\hline U.S. AgBank, FCB & 2006 & 1.000 & 4 & Valley ACA & 2006 & 0.921 & 4 \\
\hline AgCredit of South Texas ACA & 2005 & 0.827 & 2 & Puerto Rico ACA & 2005 & 0.556 & 3 \\
\hline AgCredit of South Texas ACA & 2006 & 0.959 & 4 & Puerto Rico ACA & 2006 & 0.742 & 1 \\
\hline Louisiana Ag Credit, ACA & 2005 & 1.000 & 4 & Chattanooga ACA & 2005 & 0.727 & 1 \\
\hline Louisiana Ag Credit, ACA & 2006 & 0.942 & 4 & Chattanooga ACA & 2006 & 0.825 & 2 \\
\hline First Ag Credit FCS & 2005 & 0.752 & 1 & Cape Fear ACA & 2005 & 0.579 & 3 \\
\hline First Ag Credit FCS & 2006 & 1.000 & 4 & Cape Fear ACA & 2006 & 0.762 & 2 \\
\hline Ag New Mexico, FCS, ACA & 2005 & 0.729 & 1 & MidAtlantic ACA & 2005 & 0.675 & 1 \\
\hline Ag New Mexico, FCS, ACA & 2006 & 0.888 & 4 & MidAtlantic ACA & 2006 & 0.799 & 2 \\
\hline Texas AgFinance FCS & 2005 & 0.690 & 1 & ArborOne, ACA & 2005 & 0.643 & 3 \\
\hline Texas AgFinance FCS & 2006 & 0.929 & 4 & ArborOne, ACA & 2006 & 1.000 & 4 \\
\hline Great Plains Ag Credit, ACA & 2005 & 0.612 & 3 & Colonial ACA & 2005 & 0.612 & 3 \\
\hline Great Plains Ag Credit, ACA & 2006 & 0.770 & 2 & Colonial ACA & 2006 & 0.741 & 1 \\
\hline AgriLand FCS & 2005 & 0.718 & 1 & Southwest Georgia ACA & 2005 & 0.587 & 3 \\
\hline AgriLand FCS & 2006 & 0.868 & 2 & Southwest Georgia ACA & 2006 & 0.903 & 4 \\
\hline
\end{tabular}


Table 2

Efficiency Measure Prior (2005-2006) (Continued)

\begin{tabular}{|c|c|c|c|c|c|c|c|}
\hline Name of the Bank & Year & $\begin{array}{c}\text { Efficiency } \\
\text { Scores }\end{array}$ & $\begin{array}{l}\text { Cluster } \\
\text { group }\end{array}$ & Name of the Bank & Year & $\begin{array}{c}\text { Efficiency } \\
\text { Scores }\end{array}$ & Cluster group \\
\hline Capital Farm Credit ACA & 2005 & 0.700 & 1 & AgChoice ACA & 2005 & 0.703 & 1 \\
\hline Capital Farm Credit ACA & 2006 & 1.000 & 4 & AgChoice ACA & 2006 & 0.838 & 2 \\
\hline AgTexas FCS & 2005 & 0.840 & 2 & Northwest Florida ACA & 2005 & 0.567 & 3 \\
\hline AgTexas FCS & 2006 & 1.000 & 4 & Northwest Florida ACA & 2006 & 0.738 & 1 \\
\hline Southwest Texas ACA & 2005 & 0.669 & 1 & South Florida ACA & 2005 & 0.582 & 3 \\
\hline Southwest Texas ACA & 2006 & 0.990 & 4 & South Florida ACA & 2006 & 0.668 & 1 \\
\hline Central Texas ACA & 2006 & 0.890 & 4 & Central Florida ACA & 2005 & 0.597 & 3 \\
\hline Heritage Land Bank, ACA & 2005 & 0.750 & 1 & Central Florida ACA & 2006 & 0.745 & 1 \\
\hline Heritage Land Bank, ACA & 2006 & 0.896 & 4 & North Florida ACA & 2005 & 0.608 & 3 \\
\hline Lone Star, ACA & 2005 & 0.805 & 2 & North Florida ACA & 2006 & 0.774 & 2 \\
\hline Lone Star, ACA & 2006 & 1.000 & 4 & FC of the Virginias ACA & 2005 & 0.703 & 1 \\
\hline Southwest Florida ACA & 2005 & 0.594 & 3 & $\mathrm{FC}$ of the Virginias ACA & 2006 & 0.823 & 2 \\
\hline Southwest Florida ACA & 2006 & 0.806 & 2 & Carolina ACA & 2005 & 0.776 & 2 \\
\hline Carolina ACA & 2005 & 0.776 & 2 & Carolina ACA & 2006 & 0.956 & 4 \\
\hline Carolina ACA & 2006 & 0.956 & 4 & AgCarolina ACA & 2005 & 0.627 & 3 \\
\hline AgCarolina ACA & 2005 & 0.627 & 3 & AgCarolina ACA & 2006 & 0.868 & 2 \\
\hline AgCarolina ACA & 2006 & 0.868 & 2 & AgGeorgia ACA & 2005 & 0.675 & 1 \\
\hline AgGeorgia ACA & 2005 & 0.675 & 1 & AgGeorgia ACA & 2006 & 0.816 & 2 \\
\hline AgGeorgia ACA & 2006 & 0.816 & 2 & AgSouth ACA & 2005 & 0.776 & 2 \\
\hline AgSouth ACA & 2005 & 0.776 & 2 & AgSouth ACA & 2006 & 0.910 & 4 \\
\hline AgSouth ACA & 2006 & 0.910 & 4 & Jackson Purchase ACA & 2005 & 0.668 & 1 \\
\hline Jackson Purchase ACA & 2005 & 0.668 & 1 & Jackson Purchase ACA & 2006 & 0.848 & 2 \\
\hline Jackson Purchase ACA & 2006 & 0.848 & 2 & Western Arkansas ACA & 2005 & 0.622 & 3 \\
\hline Grand Forks ACA & 2005 & 0.534 & 3 & Western Arkansas ACA & 2006 & 0.774 & 2 \\
\hline Grand Forks ACA & 2006 & 0.724 & 1 & Badgerland ACA & 2005 & 0.601 & 3 \\
\hline Mandan ACA & 2005 & 0.610 & 3 & Badgerland ACA & 2006 & 0.815 & 2 \\
\hline Mandan ACA & 2006 & 0.738 & 1 & AgHeritage ACA & 2005 & 0.615 & 3 \\
\hline FCS of Illinois ACA & 2005 & 0.629 & 3 & AgHeritage ACA & 2006 & 0.739 & 1 \\
\hline FCS of Illinois ACA & 2006 & 0.803 & 2 & AgCountry ACA & 2005 & 0.631 & 3 \\
\hline FCS of America ACA & 2005 & 0.779 & 2 & AgCountry ACA & 2006 & 0.825 & 2 \\
\hline FCS of America ACA & 2006 & 0.918 & 4 & Progressive FCS, ACA & 2005 & 0.571 & 3 \\
\hline Midsouth ACA & 2005 & 0.597 & 3 & Progressive FCS, ACA & 2006 & 0.733 & 1 \\
\hline Midsouth ACA & 2006 & 0.711 & 1 & Mid-America ACA & 2005 & 0.700 & 1 \\
\hline 1st Farm Credit Services, ACA & 2005 & 0.595 & 3 & Mid-America ACA & 2006 & 0.804 & 2 \\
\hline 1st Farm Credit Services, ACA & 2006 & 0.774 & 2 & Maine ACA & 2006 & 0.873 & 2 \\
\hline United ACA & 2005 & 0.599 & 3 & Yankee ACA & 2006 & 0.767 & 2 \\
\hline United ACA & 2006 & 0.785 & 2 & Western New York ACA & 2005 & 0.490 & 3 \\
\hline FCS Financial, ACA & 2005 & 0.687 & 1 & First Pioneer ACA & 2005 & 0.682 & 1 \\
\hline FCS Financial, ACA & 2006 & 0.825 & 2 & & & & \\
\hline
\end{tabular}

The calculated efficiency measures vary from 0.490 to 1.000 . The input-oriented efficiency analysis provides information on how much the bank should increase the level of inputs of an inefficient bank to become DEA-efficient whilst keeping the current level of output fixed. In Tables 2, the cluster group column, 1 indicates inefficient banks (I), 2 indicates efficient bank (E), 3 indicates highly inefficient banks (HI), and 4 indicates highly efficient banks (HE). For example, Table 2 shows that the bank FCB of Texas is highly efficient in 2005, but in 2006, its efficiency measure decreased from 1.000 in 2005 to 0.951 in 2006. After classifying the efficiency measures into four clusters, Table 3 shows the accuracy, confidence interval, and the parameters associated with the different kernel functions of SVM.

Table 3

Performance criteria from 2005 to 2006

\begin{tabular}{lllll}
\hline & Linear & RBF & Sigmoid & Polynomial \\
\hline Accuracy & 0.930 & 0.972 & 0.971 & 0.860 \\
Error & 0.170 & 0.28 & 0.29 & 0.240 \\
$95 \%$ CI & $0.840,0.963$ & $0.857,0.993$ & $0.854,0.990$ & $0.80,0.93$ \\
$\mathrm{a}$ & & & 1.683 & 0.444 \\
$\gamma$ & & 4.463 & 0.951 & 3 \\
\hline
\end{tabular}


The basic SVM framework is designed to determine the optimal decision boundary. To obtain an unbiased performance estimate, cross-validation was performed (See Table 4) with a total of 36 banks in the testing data set comprised of 13 inefficient banks, 8 efficient banks, 11 highly inefficient banks and 4 highly efficient banks. While applying the RBF kernel, 12 banks were correctly classified as inefficient, 8 banks were correctly classified as efficient, 11 banks were correctly classified as highly inefficient, and 4 banks were correctly classified as highly efficient. Using the linear kernel function, 13 banks were correctly classified as inefficient, 8 banks were correctly classified as efficient, 10 banks were correctly classified as highly inefficient, and 3 banks were correctly classified as highly efficient. With the polynomial kernel function, 10 banks were correctly classified as inefficient, 7 banks were correctly classified as efficient, 10 banks were correctly classified as highly inefficient, and 4 banks were correctly classified as highly efficient. When applying the sigmoid kernel function, 12 banks were correctly classified as inefficient, 8 banks were correctly classified as efficient, 11 banks were correctly classified as highly inefficient, and 4 banks were correctly classified as highly efficient.

\subsection{During the financial crisis}

Tables 4 presents a summary of the efficiency measures of banks in our sample by year. Column 2 gives the year of the technical efficiency for each individual bank, followed by technical efficiency measures in column 3. Table 4 provides the efficiency measures that changed on the year basis. The results of our analysis show that there was a big fluctuation in the efficiency scores. In column 4 of Table 4, the cluster group of the technical efficiency measure is presented in which 1 indicates highly inefficient banks (HI), 2 indicates highly efficient bank (HE), 3 indicates efficient banks (E), and 4 indicates inefficient banks (I). Additionally, Table 4 shows the cluster group of the individual bank is changing. This is for example seen with the bank of FCB of Texas. Table 8 shows the accuracy, confidence interval, and the parameters associated with the different kernel functions of SVM.

Table 4

Efficiency Measure (2007-2009)

\begin{tabular}{|c|c|c|c|c|c|c|c|}
\hline Name of the Bank & Year & Efficiency & Cluster group & Name of the Bank & Year & Efficiency & Cluster group \\
\hline FCB of Texas & 2007 & 1 & 2 & AgCountry ACA & 2009 & 0.526 & 4 \\
\hline FCB of Texas & 2008 & 0.797 & 3 & ArborOne, ACA & 2007 & 1 & 2 \\
\hline FCB of Texas & 2009 & 0.596 & 1 & ArborOne, ACA & 2008 & 1 & 2 \\
\hline AgFirst FCB & 2007 & 1 & 2 & ArborOne, ACA & 2009 & 0.912 & 2 \\
\hline AgFirst FCB & 2008 & 0.744 & 3 & Colonial ACA & 2007 & 0.77 & 3 \\
\hline AgFirst FCB & 2009 & 0.446 & 4 & Colonial ACA & 2008 & 0.682 & 1 \\
\hline AgriBank, FCB & 2007 & 1 & 2 & Colonial ACA & 2009 & 0.603 & 1 \\
\hline AgriBank, FCB & 2008 & 0.683 & 1 & MidAtlantic ACA & 2009 & 0.668 & 1 \\
\hline AgriBank, FCB & 2009 & 0.42 & 4 & Southwest Georgia & 2007 & 0.965 & 2 \\
\hline U.S. AgBank, FCB & 2007 & 0.967 & 2 & Southwest Georgia & 2008 & 0.798 & 3 \\
\hline U.S. AgBank, FCB & 2008 & 0.693 & 1 & Southwest Georgia & 2009 & 0.8 & 3 \\
\hline U.S. AgBank, FCB & 2009 & 0.459 & 4 & AgChoice ACA & 2007 & 0.856 & 3 \\
\hline AgCredit of South Texas ACA & 2007 & 1 & 2 & AgChoice ACA & 2008 & 0.714 & 1 \\
\hline AgCredit of South Texas ACA & 2008 & 0.992 & 2 & AgChoice ACA & 2009 & 0.608 & 1 \\
\hline AgCredit of South Texas ACA & 2009 & 0.825 & 3 & Northwest Florida ACA & 2007 & 0.757 & 3 \\
\hline Louisiana Ag Credit, ACA & 2007 & 0.871 & 3 & Northwest Florida ACA & 2008 & 0.648 & 1 \\
\hline Louisiana Ag Credit, ACA & 2008 & 0.887 & 3 & Northwest Florida ACA & 2009 & 0.631 & 1 \\
\hline Louisiana Ag Credit, ACA & 2009 & 0.825 & 3 & South Florida ACA & 2007 & 0.737 & 3 \\
\hline First Ag Credit FCS & 2007 & 1 & 2 & South Florida ACA & 2008 & 0.617 & 1 \\
\hline First Ag Credit FCS & 2008 & 0.819 & 3 & South Florida ACA & 2009 & 0.514 & 4 \\
\hline Ag New Mexico, FCS, ACA & 2007 & 0.96 & 2 & Central Florida ACA & 2007 & 0.858 & 3 \\
\hline Ag New Mexico, FCS, ACA & 2008 & 0.769 & 3 & Central Florida ACA & 2008 & 0.725 & 1 \\
\hline Ag New Mexico, FCS, ACA & 2009 & 0.621 & 1 & Central Florida ACA & 2009 & 0.614 & 1 \\
\hline Texas AgFinance FCS & 2007 & 1 & 2 & North Florida ACA & 2007 & 0.826 & 3 \\
\hline Texas AgFinance FCS & 2008 & 0.793 & 3 & North Florida ACA & 2008 & 0.692 & 1 \\
\hline Texas AgFinance FCS & 2009 & 0.651 & 1 & North Florida ACA & 2009 & 0.614 & 1 \\
\hline Great Plains Ag Credit, ACA & 2007 & 0.812 & 3 & Southwest Florida ACA & 2007 & 0.971 & 2 \\
\hline Great Plains Ag Credit, ACA & 2008 & 0.621 & 1 & Southwest Florida ACA & 2008 & 0.841 & 3 \\
\hline Great Plains Ag Credit, ACA & 2009 & 0.489 & 4 & Southwest Florida ACA & 2009 & 0.756 & 3 \\
\hline AgriLand FCS & 2007 & 0.881 & 3 & FC of the Virginias & 2007 & 0.838 & 3 \\
\hline AgriLand FCS & 2008 & 0.745 & 3 & FC of the Virginias & 2008 & 0.789 & 3 \\
\hline AgriLand FCS & 2009 & 0.664 & 1 & FC of the Virginias & 2009 & 0.744 & 3 \\
\hline
\end{tabular}


Table 4

Efficiency Measure (2007-2009) (Continued)

\begin{tabular}{|c|c|c|c|c|c|c|c|}
\hline Name of the Bank & Year & Efficiency & Cluster group & Name of the Bank & Year & Efficiency & Cluster group \\
\hline AgTexas FCS & 2007 & 1 & 2 & Carolina ACA & 2007 & 0.929 & 2 \\
\hline AgTexas FCS & 2008 & 0.853 & 3 & Carolina ACA & 2008 & 0.794 & 3 \\
\hline AgTexas FCS & 2009 & 0.847 & 3 & Carolina ACA & 2009 & 0.703 & 1 \\
\hline Capital Farm Credit ACA & 2007 & 1 & 2 & AgCarolina ACA & 2007 & 0.857 & 3 \\
\hline Capital Farm Credit ACA & 2008 & 0.833 & 3 & AgCarolina ACA & 2008 & 0.714 & 1 \\
\hline Central Texas ACA & 2007 & 0.985 & 2 & AgCarolina ACA & 2009 & 0.596 & 1 \\
\hline Central Texas ACA & 2008 & 0.704 & 1 & AgGeorgia ACA & 2007 & 0.821 & 3 \\
\hline Central Texas ACA & 2009 & 0.527 & 4 & AgGeorgia ACA & 2008 & 0.74 & 3 \\
\hline Heritage Land Bank, ACA & 2007 & 0.991 & 2 & AgGeorgia ACA & 2009 & 0.65 & 1 \\
\hline Heritage Land Bank, ACA & 2008 & 0.808 & 3 & AgSouth ACA & 2007 & 0.884 & 3 \\
\hline Heritage Land Bank, ACA & 2009 & 0.651 & 1 & AgSouth ACA & 2008 & 0.758 & 3 \\
\hline Capital Farm Credit, ACA & 2009 & 0.649 & 1 & AgSouth ACA & 2009 & 0.7 & 1 \\
\hline Texas Land Bank, ACA & 2007 & 0.952 & 2 & Jackson Purchase ACA & 2007 & 0.927 & 2 \\
\hline Texas Land Bank, ACA & 2008 & 0.782 & 3 & Jackson Purchase ACA & 2008 & 0.769 & 3 \\
\hline Texas Land Bank, ACA & 2009 & 0.623 & 1 & Jackson Purchase ACA & 2009 & 0.712 & 1 \\
\hline Lone Star, ACA & 2007 & 1 & 2 & AG CREDIT ACA & 2007 & 0.773 & 3 \\
\hline Lone Star, ACA & 2008 & 0.831 & 3 & AG CREDIT ACA & 2008 & 0.697 & 1 \\
\hline Lone Star, ACA & 2009 & 0.606 & 1 & AG CREDIT ACA & 2009 & 0.574 & 1 \\
\hline Legacy Ag Credit, ACA & 2007 & 1 & 2 & GreenStone ACA & 2007 & 0.769 & 3 \\
\hline Legacy Ag Credit, ACA & 2008 & 0.911 & 2 & GreenStone ACA & 2008 & 0.674 & 1 \\
\hline Legacy Ag Credit, ACA & 2009 & 0.794 & 3 & GreenStone ACA & 2009 & 0.509 & 4 \\
\hline Southern AgCredit, ACA & 2009 & 0.585 & 1 & AgStar ACA & 2007 & 1 & 2 \\
\hline First South ACA & 2007 & 0.834 & 3 & AgStar ACA & 2008 & 0.834 & 3 \\
\hline First South ACA & 2008 & 0.784 & 3 & AgStar ACA & 2009 & 0.666 & 1 \\
\hline First South ACA & 2009 & 0.717 & 1 & North Dakota ACA & 2007 & 0.768 & 3 \\
\hline Central Kentucky ACA & 2007 & 1 & 2 & North Dakota ACA & 2008 & 0.575 & 1 \\
\hline Central Kentucky ACA & 2008 & 0.895 & 2 & North Dakota ACA & 2009 & 0.398 & 4 \\
\hline Central Kentucky ACA & 2009 & 0.796 & 3 & Delta ACA & 2007 & 1 & 2 \\
\hline Valley ACA & 2007 & 0.941 & 2 & Delta ACA & 2008 & 1 & 2 \\
\hline Valley ACA & 2008 & 0.86 & 3 & Delta ACA & 2009 & 0.925 & 2 \\
\hline Puerto Rico ACA & 2007 & 0.788 & 3 & Grand Forks ACA & 2007 & 0.728 & 3 \\
\hline Puerto Rico ACA & 2008 & 0.583 & 1 & Mandan ACA & 2007 & 0.784 & 3 \\
\hline Puerto Rico ACA & 2009 & 0.495 & 4 & Mandan ACA & 2008 & 0.614 & 1 \\
\hline Chattanooga ACA & 2007 & 0.869 & 3 & Mandan ACA & 2009 & 0.441 & 4 \\
\hline Chattanooga ACA & 2008 & 0.764 & 3 & FCS of Illinois ACA & 2007 & 0.834 & 3 \\
\hline Chattanooga ACA & 2009 & 0.836 & 3 & FCS of Illinois ACA & 2008 & 0.722 & 1 \\
\hline Cape Fear ACA & 2007 & 0.81 & 3 & FCS of Illinois ACA & 2009 & 0.509 & 4 \\
\hline Cape Fear ACA & 2008 & 0.69 & 1 & FCS of America ACA & 2007 & 1 & 2 \\
\hline Cape Fear ACA & 2009 & 0.594 & 1 & FCS of America ACA & 2008 & 0.862 & 3 \\
\hline Cape Fear ACA & 2009 & 0.594 & 1 & 1st Farm Credit & 2007 & 0.756 & 3 \\
\hline MidAtlantic ACA & 2007 & 0.794 & 3 & 1st Farm Credit & 2008 & 0.663 & 1 \\
\hline MidAtlantic ACA & 2008 & 0.695 & 1 & 1st Farm Credit & 2009 & 0.516 & 4 \\
\hline FCS of America ACA & 2009 & 0.656 & 1 & United ACA & 2007 & 0.812 & 3 \\
\hline Midsouth ACA & 2007 & 0.74 & 3 & United ACA & 2008 & 0.655 & 1 \\
\hline Midsouth ACA & 2008 & 0.583 & 1 & United ACA & 2009 & 0.481 & 4 \\
\hline Midsouth ACA & 2009 & 0.46 & 4 & FCS Financial, ACA & 2007 & 0.848 & 3 \\
\hline Western Arkansas ACA & 2007 & 0.804 & 3 & FCS Financial, ACA & 2008 & 0.748 & 3 \\
\hline Western Arkansas ACA & 2008 & 0.693 & 1 & FCS Financial, ACA & 2009 & 0.525 & 4 \\
\hline Western Arkansas ACA & 2009 & 0.53 & 4 & Mid-America ACA & 2007 & 0.765 & 3 \\
\hline Badgerland ACA & 2007 & 0.815 & 3 & Mid-America ACA & 2008 & 0.762 & 3 \\
\hline Badgerland ACA & 2008 & 0.637 & 1 & Mid-America ACA & 2009 & 0.656 & 1 \\
\hline Badgerland ACA & 2009 & 0.42 & 4 & Maine ACA & 2007 & 0.938 & 2 \\
\hline AgHeritage ACA & 2007 & 0.78 & 3 & Maine ACA & 2008 & 0.667 & 1 \\
\hline AgHeritage ACA & 2008 & 0.703 & 1 & Yankee ACA & 2008 & 0.594 & 1 \\
\hline AgHeritage ACA & 2009 & 0.504 & 4 & Western New York & 2008 & 0.432 & 4 \\
\hline AgCountry ACA & 2007 & 0.79 & 3 & Western New York & 2009 & 0.314 & 4 \\
\hline Progressive FCS, ACA & 2007 & 0.784 & 3 & First Pioneer ACA & 2008 & 0.541 & 4 \\
\hline Progressive FCS, ACA & 2008 & 0.59 & 1 & First Pioneer ACA & 2009 & 0.388 & 4 \\
\hline Progressive FCS, ACA & 2009 & 0.427 & 4 & American AgCredit & 2009 & 0.539 & 4 \\
\hline AgCountry ACA & 2008 & 0.638 & 1 & & & & \\
\hline
\end{tabular}

During 2007-2009, only 54 banks (6 inefficient banks, 20 efficient banks, 17 highly inefficient banks and 11 highly efficient banks) were considered in the testing data set. Using the RBF kernel, to validate whether the training was efficient, 15 banks were correctly classified as highly inefficient, 8 banks were correctly classified as highly efficient, 19 banks were correctly classified as efficient, and 6 banks were correctly classified as inefficient. For the linear kernel, 16 banks were correctly classified as highly 
inefficient, 9 banks were correctly classified as highly efficient, 20 banks were correctly classified as efficient and 6 banks were correctly classified as inefficient. Using the polynomial kernel, 12 banks were correctly classified as highly inefficient, 10 were correctly classified as highly efficient, 19 banks were correctly classified as efficient, and 6 banks were correctly classified as inefficient. Using the sigmoid kernel, 16 banks were correctly classified as highly inefficient, 8 were correctly classified as highly efficient, 18 banks were correctly classified as efficient, and 5 banks were correctly classified as inefficient.

\section{Table 5}

Performance criteria from 2007-2009

\begin{tabular}{lllll}
\hline & Linear & RBF & Sigmoid & Polynomial \\
\hline Accuracy & 0.944 & 0.899 & 0.870 & 0.850 \\
Error & 0.56 & 0.111 & 0.130 & 0.150 \\
$95 \%$ CI & $0.846,0.988$ & $0.774,0.958$ & $0.751,0.946$ & $0.751,0.946$ \\
a & & & 1.683 & 0.446 \\
$\gamma$ & & 4.43 & 0.950 & 3 \\
\hline
\end{tabular}

\subsection{After the financial crisis of 2007-2009}

Tables 9-13 present the efficiency measures for 2010-2016 using the input oriented BCC model. The stationary test of the efficiency measures was conducted and resulted in not having enough evidence to reject the null hypothesis of stationarity at $1 \%$. In Table 6 , four columns are present: 1 ) The bank name; 2) The year of the estimated efficiency measures; 3) The estimated efficiency measure and 4) The cluster group of the efficiency measure. While accounting for the cluster group in Tables 9-13, 1 indicates highly efficient banks (HE), 2 indicates efficient bank (E), 3 indicates highly inefficient banks (HI), and 4 indicates inefficient banks (I). To observe the impact of DEA measure on the bank performance, Table 14 shows the accuracy, confidence interval, and the parameters associated with the different kernel functions of SVM.

Table 6

Efficiency Measure (2010-2016)

\begin{tabular}{|c|c|c|c|c|c|c|c|}
\hline Name of the Bank & Year & $\begin{array}{c}\text { Efficiency } \\
\text { Scores }\end{array}$ & $\begin{array}{c}\text { Cluster } \\
\text { group }\end{array}$ & Name of the Bank & Year & $\begin{array}{c}\text { Efficiency } \\
\text { Scores }\end{array}$ & Cluster group \\
\hline FCB of Texas & 2010 & 0.975 & 1 & Texas Land Bank, ACA & 2010 & 0.844 & 2 \\
\hline FCB of Texas & 2011 & 0.731 & 2 & Texas Land Bank, ACA & 2011 & 0.712 & 3 \\
\hline FCB of Texas & 2012 & 0.618 & 3 & Texas Land Bank, ACA & 2012 & 0.611 & 3 \\
\hline FCB of Texas & 2013 & 0.539 & 4 & Texas Land Bank, ACA & 2013 & 0.594 & 3 \\
\hline FCB of Texas & 2015 & 0.575 & 4 & Lone Star, ACA & 2010 & 0.866 & 2 \\
\hline FCB of Texas & 2016 & 0.672 & 3 & Lone Star, ACA & 2011 & 0.815 & 2 \\
\hline AgFirst FCB & 2010 & 0.733 & 2 & Lone Star, ACA & 2012 & 0.739 & 2 \\
\hline AgFirst FCB & 2011 & 0.572 & 4 & Lone Star, ACA & 2013 & 0.638 & 3 \\
\hline AgFirst FCB & 2012 & 0.429 & 4 & Legacy Ag Credit, ACA & 2010 & 0.916 & 1 \\
\hline AgFirst FCB & 2013 & 0.408 & 4 & Legacy Ag Credit, ACA & 2011 & 0.992 & 1 \\
\hline AgFirst FCB & 2015 & 0.518 & 4 & Legacy Ag Credit, ACA & 2012 & 1 & 1 \\
\hline AgFirst FCB & 2016 & 0.643 & 3 & Legacy Ag Credit, ACA & 2013 & 1 & 1 \\
\hline AgriBank, FCB & 2010 & 1 & 1 & Legacy Ag Credit, ACA & 2015 & 1 & 1 \\
\hline AgriBank, FCB & 2011 & 0.958 & 1 & Legacy Ag Credit, ACA & 2016 & 0.967 & 1 \\
\hline AgriBank, FCB & 2012 & 0.812 & 2 & Louisiana Land Bank, & 2010 & 0.816 & 2 \\
\hline AgriBank, FCB & 2013 & 0.715 & 3 & Louisiana Land Bank, & 2011 & 0.718 & 3 \\
\hline AgriBank, FCB & 2015 & 0.958 & 1 & Louisiana Land Bank, & 2012 & 0.819 & 2 \\
\hline AgriBank, FCB & 2016 & 1 & 1 & Louisiana Land Bank, & 2013 & 0.707 & 3 \\
\hline U.S. AgBank, FCB & 2010 & 0.814 & 2 & Louisiana Land Bank, & 2015 & 0.753 & 2 \\
\hline U.S. AgBank, FCB & 2011 & 0.621 & 3 & Louisiana Land Bank, & 2016 & 0.714 & 3 \\
\hline AgCredit of South Texas ACA & 2010 & 1 & 1 & Mississippi Land Bank, & 2010 & 0.839 & 2 \\
\hline Louisiana Ag Credit, ACA & 2010 & 0.976 & 1 & Mississippi Land Bank, & 2011 & 0.734 & 2 \\
\hline Ag New Mexico, FCS, ACA & 2010 & 0.901 & 1 & Mississippi Land Bank, & 2012 & 0.683 & 3 \\
\hline Ag New Mexico, FCS, ACA & 2011 & 0.86 & 2 & Mississippi Land Bank, & 2013 & 0.64 & 3 \\
\hline Ag New Mexico, FCS, ACA & 2012 & 0.816 & 2 & Mississippi Land Bank, & 2015 & 0.675 & 3 \\
\hline Ag New Mexico, FCS, ACA & 2013 & 0.775 & 2 & Mississippi Land Bank, & 2016 & 0.67 & 3 \\
\hline Ag New Mexico, FCS, ACA & 2015 & 0.889 & 1 & Southern AgCredit, ACA & 2010 & 0.792 & 2 \\
\hline
\end{tabular}


Table 6

Efficiency Measure (2010-2016)

\begin{tabular}{|c|c|c|c|c|c|c|c|}
\hline Name of the Bank & Year & $\begin{array}{c}\text { Efficiency } \\
\text { Scores }\end{array}$ & $\begin{array}{l}\text { Cluster } \\
\text { group }\end{array}$ & Name of the Bank & Year & $\begin{array}{c}\text { Efficiency } \\
\text { Scores }\end{array}$ & Cluster group \\
\hline Ag New Mexico, FCS, ACA & 2016 & 0.846 & 2 & Southern AgCredit, ACA & 2011 & 0.682 & 3 \\
\hline Texas AgFinance FCS & 2010 & 0.883 & 1 & Southern AgCredit, ACA & 2012 & 0.607 & 3 \\
\hline Great Plains Ag Credit, ACA & 2010 & 0.569 & 4 & Southern AgCredit, ACA & 2013 & 0.565 & 4 \\
\hline Great Plains Ag Credit, ACA & 2011 & 0.521 & 4 & Southern AgCredit, ACA & 2015 & 0.567 & 4 \\
\hline Great Plains Ag Credit, ACA & 2012 & 0.504 & 4 & Southern AgCredit, ACA & 2016 & 0.613 & 3 \\
\hline Great Plains Ag Credit, ACA & 2013 & 0.489 & 4 & Alabama ACA & 2010 & 0.907 & 1 \\
\hline AgriLand FCS & 2010 & 0.739 & 2 & Alabama ACA & 2011 & 0.763 & 2 \\
\hline AgriLand FCS & 2011 & 0.726 & 2 & Alabama ACA & 2012 & 0.692 & 3 \\
\hline AgriLand FCS & 2012 & 0.71 & 3 & Alabama ACA & 2013 & 0.645 & 3 \\
\hline AgriLand FCS & 2013 & 0.738 & 2 & Alabama ACA & 2015 & 0.683 & 3 \\
\hline Texas AgFinance FCS & 2011 & 0.639 & 3 & Alabama ACA & 2016 & 0.73 & 2 \\
\hline Texas AgFinance FCS & 2012 & 0.7 & 3 & Alabama Ag Credit, ACA & 2010 & 0.799 & 2 \\
\hline Texas AgFinance FCS & 2013 & 0.634 & 3 & Alabama Ag Credit, ACA & 2011 & 0.712 & 3 \\
\hline AgTexas FCS & 2010 & 1 & 1 & Alabama Ag Credit, ACA & 2012 & 0.688 & 3 \\
\hline AgTexas FCS & 2011 & 0.981 & 1 & Alabama Ag Credit, ACA & 2013 & 0.652 & 3 \\
\hline AgTexas FCS & 2012 & 0.779 & 2 & Alabama Ag Credit, ACA & 2015 & 0.698 & 3 \\
\hline AgTexas FCS & 2013 & 0.688 & 3 & Alabama Ag Credit, ACA & 2016 & 0.709 & 3 \\
\hline Texas FCS & 2015 & 0.768 & 2 & First South ACA & 2010 & 1 & 1 \\
\hline Texas FCS & 2016 & 0.72 & 3 & First South ACA & 2011 & 0.978 & 1 \\
\hline AgTexas FCS & 2015 & 0.737 & 2 & First South ACA & 2012 & 0.924 & 1 \\
\hline AgTexas FCS & 2016 & 0.726 & 2 & First South ACA & 2013 & 0.934 & 1 \\
\hline Lone Star, ACA & 2015 & 0.711 & 3 & First South ACA & 2015 & 0.979 & 1 \\
\hline Lone Star, ACA & 2016 & 0.705 & 3 & First South ACA & 2016 & 1 & 1 \\
\hline Central Texas ACA & 2010 & 0.703 & 3 & Central Kentucky ACA & 2010 & 0.918 & 1 \\
\hline Central Texas ACA & 2011 & 0.648 & 3 & Central Kentucky ACA & 2011 & 0.79 & 2 \\
\hline Central Texas ACA & 2012 & 0.601 & 3 & Central Kentucky ACA & 2012 & 0.727 & 2 \\
\hline Central Texas ACA & 2013 & 0.585 & 4 & Central Kentucky ACA & 2013 & 0.689 & 3 \\
\hline Central Texas ACA & 2015 & 0.663 & 3 & Central Kentucky ACA & 2015 & 0.687 & 3 \\
\hline Central Texas ACA & 2016 & 0.816 & 2 & Central Kentucky ACA & 2016 & 0.655 & 3 \\
\hline Heritage Land Bank, ACA & 2010 & 0.889 & 1 & Puerto Rico ACA & 2010 & 0.621 & 3 \\
\hline Heritage Land Bank, ACA & 2011 & 0.903 & 1 & Puerto Rico ACA & 2011 & 0.644 & 3 \\
\hline Heritage Land Bank, ACA & 2012 & 0.845 & 2 & Puerto Rico ACA & 2012 & 0.682 & 3 \\
\hline Heritage Land Bank, ACA & 2013 & 0.837 & 2 & Puerto Rico ACA & 2013 & 0.674 & 3 \\
\hline Heritage Land Bank, ACA & 2015 & 0.965 & 1 & Puerto Rico ACA & 2015 & 0.778 & 2 \\
\hline Heritage Land Bank, ACA & 2016 & 0.923 & 1 & Puerto Rico ACA & 2016 & 0.828 & 2 \\
\hline Capital Farm Credit, ACA & 2010 & 1 & 1 & Chattanooga ACA & 2010 & 1 & 1 \\
\hline Capital Farm Credit, ACA & 2011 & 0.865 & 2 & Chattanooga ACA & 2011 & 0.946 & 1 \\
\hline Capital Farm Credit, ACA & 2012 & 0.761 & 2 & Chattanooga ACA & 2012 & 0.924 & 1 \\
\hline Capital Farm Credit, ACA & 2013 & 0.679 & 3 & Cape Fear ACA & 2010 & 0.713 & 3 \\
\hline Capital Farm Credit, ACA & 2015 & 0.769 & 2 & Cape Fear ACA & 2011 & 0.618 & 3 \\
\hline Capital Farm Credit, ACA & 2016 & 0.811 & 2 & Cape Fear ACA & 2012 & 0.599 & 3 \\
\hline Cape Fear ACA & 2013 & 0.593 & 3 & AgCarolina ACA & 2010 & 0.748 & 2 \\
\hline Cape Fear ACA & 2015 & 0.636 & 3 & AgCarolina ACA & 2011 & 0.686 & 3 \\
\hline Cape Fear ACA & 2016 & 0.683 & 3 & AgCarolina ACA & 2012 & 0.649 & 3 \\
\hline ArborOne, ACA & 2010 & 1 & 1 & AgCarolina ACA & 2013 & 0.613 & 3 \\
\hline ArborOne, ACA & 2011 & 0.979 & 1 & AgCarolina ACA & 2015 & 0.616 & 3 \\
\hline ArborOne, ACA & 2012 & 0.914 & 1 & AgCarolina ACA & 2016 & 0.646 & 3 \\
\hline ArborOne, ACA & 2013 & 0.757 & 2 & AgGeorgia ACA & 2010 & 0.889 & 1 \\
\hline ArborOne, ACA & 2015 & 0.698 & 3 & AgGeorgia ACA & 2011 & 0.814 & 2 \\
\hline ArborOne, ACA & 2016 & 0.681 & 3 & AgGeorgia ACA & 2012 & 0.784 & 2 \\
\hline Colonial ACA & 2010 & 0.651 & 3 & AgGeorgia ACA & 2013 & 0.741 & 2 \\
\hline Colonial ACA & 2011 & 0.617 & 3 & AgGeorgia ACA & 2015 & 0.743 & 2 \\
\hline Colonial ACA & 2012 & 0.599 & 3 & AgGeorgia ACA & 2016 & 0.755 & 2 \\
\hline Colonial ACA & 2013 & 0.619 & 3 & Florida ACA & 2011 & 0.741 & 2 \\
\hline Colonial ACA & 2015 & 0.694 & 3 & Florida ACA & 2012 & 0.793 & 2 \\
\hline Colonial ACA & 2016 & 0.741 & 2 & Florida ACA & 2013 & 0.859 & 2 \\
\hline MidAtlantic ACA & 2010 & 0.959 & 1 & Florida ACA & 2015 & 0.708 & 3 \\
\hline MidAtlantic ACA & 2011 & 0.855 & 2 & Florida ACA & 2016 & 0.73 & 2 \\
\hline MidAtlantic ACA & 2012 & 0.807 & 2 & AgSouth ACA & 2010 & 0.974 & 1 \\
\hline MidAtlantic ACA & 2013 & 0.763 & 2 & AgSouth ACA & 2011 & 0.958 & 1 \\
\hline MidAtlantic ACA & 2015 & 0.818 & 2 & AgSouth ACA & 2012 & 0.901 & 1 \\
\hline MidAtlantic ACA & 2016 & 0.848 & 2 & AgSouth ACA & 2013 & 0.915 & 1 \\
\hline Southwest Georgia ACA & 2010 & 0.818 & 2 & AgSouth ACA & 2015 & 0.962 & 1 \\
\hline Southwest Georgia ACA & 2011 & 0.777 & 2 & AgSouth ACA & 2016 & 0.995 & 1 \\
\hline Southwest Georgia ACA & 2012 & 0.723 & 3 & Jackson Purchase ACA & 2010 & 0.87 & 2 \\
\hline Southwest Georgia ACA & 2013 & 0.659 & 3 & Jackson Purchase ACA & 2011 & 0.825 & 2 \\
\hline Southwest Georgia ACA & 2015 & 0.721 & 3 & Jackson Purchase ACA & 2012 & 0.811 & 2 \\
\hline
\end{tabular}


Table 6

Efficiency Measure (2010-2016) (Continued)

\begin{tabular}{|c|c|c|c|c|c|c|c|}
\hline Name of the Bank & Year & $\begin{array}{l}\text { Efficiency } \\
\text { Scores }\end{array}$ & Cluster group & Name of the Bank & Year & $\begin{array}{l}\text { Efficiency } \\
\text { Scores }\end{array}$ & $\begin{array}{l}\text { Cluster } \\
\text { group }\end{array}$ \\
\hline Southwest Georgia ACA & 2016 & 0.775 & 2 & AG CREDIT ACA & 2010 & 0.751 & 2 \\
\hline AgChoice ACA & 2010 & 0.813 & 2 & AG CREDIT ACA & 2011 & 0.733 & 2 \\
\hline AgChoice ACA & 2011 & 0.771 & 2 & AG CREDIT ACA & 2012 & 0.694 & 3 \\
\hline AgChoice ACA & 2012 & 0.751 & 2 & AG CREDIT ACA & 2013 & 0.654 & 3 \\
\hline AgChoice ACA & 2013 & 0.709 & 3 & AG CREDIT ACA & 2015 & 0.655 & 3 \\
\hline AgChoice ACA & 2015 & 0.743 & 2 & AG CREDIT ACA & 2016 & 0.685 & 3 \\
\hline AgChoice ACA & 2016 & 0.753 & 2 & River Valley AgCredit, ACA & 2012 & 0.897 & 1 \\
\hline Northwest Florida ACA & 2010 & 0.74 & 2 & River Valley AgCredit, ACA & 2013 & 0.866 & 2 \\
\hline Northwest Florida ACA & 2011 & 0.704 & 3 & River Valley AgCredit, ACA & 2015 & 0.864 & 2 \\
\hline Northwest Florida ACA & 2012 & 0.688 & 3 & River Valley AgCredit, ACA & 2016 & 0.73 & 2 \\
\hline Northwest Florida ACA & 2013 & 0.686 & 3 & GreenStone ACA & 2010 & 0.76 & 2 \\
\hline Northwest Florida ACA & 2015 & 0.64 & 3 & GreenStone ACA & 2011 & 0.704 & 3 \\
\hline Northwest Florida ACA & 2016 & 0.68 & 3 & GreenStone ACA & 2012 & 0.664 & 3 \\
\hline South Florida ACA & 2010 & 0.547 & 4 & GreenStone ACA & 2013 & 0.63 & 3 \\
\hline Central Florida ACA & 2010 & 0.621 & 3 & GreenStone ACA & 2015 & 0.692 & 3 \\
\hline Central Florida ACA & 2011 & 0.622 & 3 & GreenStone ACA & 2016 & 0.699 & 3 \\
\hline Central Florida ACA & 2012 & 0.615 & 3 & AgStar ACA & 2010 & 1 & 1 \\
\hline Central Florida ACA & 2013 & 0.611 & 3 & AgStar ACA & 2011 & 0.936 & 1 \\
\hline Central Florida ACA & 2015 & 0.621 & 3 & AgStar ACA & 2012 & 0.88 & 1 \\
\hline Central Florida ACA & 2016 & 0.639 & 3 & AgStar ACA & 2013 & 0.784 & 2 \\
\hline North Florida ACA & 2010 & 0.698 & 3 & AgStar ACA & 2015 & 0.773 & 2 \\
\hline Southwest Florida ACA & 2010 & 0.977 & 1 & AgStar ACA & 2016 & 0.823 & 2 \\
\hline FC of the Virginias ACA & 2010 & 1 & 1 & North Dakota ACA & 2010 & 0.479 & 4 \\
\hline FC of the Virginias ACA & 2011 & 0.947 & 1 & North Dakota ACA & 2011 & 0.483 & 4 \\
\hline FC of the Virginias ACA & 2012 & 0.873 & 1 & North Dakota ACA & 2012 & 0.488 & 4 \\
\hline FC of the Virginias ACA & 2013 & 0.813 & 2 & North Dakota ACA & 2013 & 0.449 & 4 \\
\hline FC of the Virginias ACA & 2015 & 0.808 & 2 & North Dakota ACA & 2015 & 0.503 & 4 \\
\hline $\mathrm{FC}$ of the Virginias ACA & 2016 & 0.83 & 2 & North Dakota ACA & 2016 & 0.586 & 4 \\
\hline Carolina ACA & 2010 & 0.976 & 1 & Delta ACA & 2010 & 1 & 1 \\
\hline Carolina ACA & 2011 & 0.925 & 1 & Delta ACA & 2011 & 0.993 & 1 \\
\hline Carolina ACA & 2012 & 0.905 & 1 & Delta ACA & 2012 & 0.999 & 1 \\
\hline Carolina ACA & 2013 & 0.872 & 1 & Delta ACA & 2013 & 1 & 1 \\
\hline Carolina ACA & 2015 & 0.849 & 2 & Delta ACA & 2015 & 0.976 & 1 \\
\hline Carolina ACA & 2016 & 0.899 & 1 & Delta ACA & 2016 & 0.969 & 1 \\
\hline Badgerland Financial ACA & 2013 & 0.559 & 4 & Farm Credit West, ACA & 2013 & 0.47 & 4 \\
\hline Badgerland Financial ACA & 2015 & 0.633 & 3 & Farm Credit West, ACA & 2015 & 0.425 & 4 \\
\hline Badgerland Financial ACA & 2016 & 0.671 & 3 & Oklahoma AgCredit, ACA & 2016 & 0.629 & 3 \\
\hline AgHeritage ACA & 2010 & 0.638 & 3 & Chisholm Trail ACA & 2012 & 0.68 & 3 \\
\hline AgHeritage ACA & 2011 & 0.6 & 3 & Chisholm Trail ACA & 2013 & 0.655 & 3 \\
\hline AgHeritage ACA & 2012 & 0.575 & 4 & Chisholm Trail ACA & 2015 & 0.667 & 3 \\
\hline AgHeritage ACA & 2013 & 0.551 & 4 & American AgCredit, ACA & 2015 & 0.804 & 2 \\
\hline AgHeritage ACA & 2015 & 0.56 & 4 & American AgCredit, ACA & 2016 & 0.866 & 2 \\
\hline AgHeritage ACA & 2016 & 0.542 & 4 & Western AgCredit, ACA & 2015 & 0.537 & 4 \\
\hline Progressive FCS, ACA & 2010 & 0.489 & 4 & Western AgCredit, ACA & 2016 & 0.522 & 4 \\
\hline Progressive FCS, ACA & 2011 & 0.478 & 4 & Farm Credit East, ACA & 2015 & 0.546 & 4 \\
\hline Progressive FCS, ACA & 2012 & 0.448 & 4 & Farm Credit East, ACA & 2016 & 0.614 & 3 \\
\hline Progressive FCS, ACA & 2013 & 0.485 & 4 & FCS Southwest ACA & 2015 & 0.48 & 4 \\
\hline Progressive FCS, ACA & 2015 & 0.491 & 4 & Western Oklahoma ACA & 2016 & 0.709 & 3 \\
\hline Progressive FCS, ACA & 2016 & 0.541 & 4 & Southwest Kansas ACA & 2016 & 0.652 & 3 \\
\hline AgCountry ACA & 2010 & 0.71 & 3 & 1st Farm Credit Services, ACA & 2010 & 0.666 & 3 \\
\hline AgCountry ACA & 2011 & 0.69 & 3 & 1st Farm Credit Services, ACA & 2011 & 0.617 & 3 \\
\hline AgCountry ACA & 2012 & 0.643 & 3 & 1st Farm Credit Services, ACA & 2012 & 0.589 & 3 \\
\hline AgCountry ACA & 2013 & 0.558 & 4 & 1st Farm Credit Services, ACA & 2013 & 0.536 & 4 \\
\hline AgCountry ACA & 2015 & 0.546 & 4 & 1st Farm Credit Services, ACA & 2015 & 0.637 & 3 \\
\hline AgCountry ACA & 2016 & 0.555 & 4 & & & & \\
\hline
\end{tabular}

To validate our model during 2010-2016, 108 banks (48 highly inefficient banks, 22 efficient banks, 15 inefficient banks, and 23 highly efficient banks) were considered in the testing data set. Using the RBF kernel, 22 banks were correctly classified as highly efficient, 22 banks were correctly classified as efficient, 45 banks were correctly classified as highly inefficient, and 15 banks were correctly classified as inefficient. For the linear kernel, 23 banks were correctly classified as highly efficient, 22 were correctly classified as efficient, 45 banks were correctly classified as highly inefficient, and 15 banks were correctly classified as inefficient. Using the polynomial kernel, 22 banks were correctly classified as highly efficient, 21 banks were correctly classified as efficient, 46 banks were correctly 
classified as highly inefficient, and 15 banks were correctly classified as inefficient. Additionally, using the sigmoid kernel, 20 banks were correctly classified as highly efficient, 14 banks were correctly classified as efficient, 43 banks were correctly classified as highly inefficient, and 15 banks were correctly classified as inefficient.

Table 7

Performance criteria from 2010-2016

\begin{tabular}{lcccc}
\hline & Linear & RBF & Sigmoid & Polynomial \\
\hline Accuracy & 0.972 & 0.963 & 0.852 & 0.960 \\
Error & 0.028 & 0.037 & 0.148 & 0.040 \\
$95 \%$ CI & $0.921,0.994$ & $0.908,0.989$ & $0.771,0.913$ & $0.910,0.989$ \\
a & & & 2.223 & 0.442 \\
$\gamma$ & & 4.428 & 0.951 & 2 \\
d & & & & \\
\hline
\end{tabular}

\section{Conclusions}

This study applies Data Envelopment Analysis (DEA) under the input oriented BCC model to measure the efficiency scores of the FCA Banks from 2005-2016 while accounting for the time dependence between the efficiency measures. The study focuses on three periods: prior to the financial crisis (20052006), during the financial crisis (2007-2009) and post the financial crisis (2010-2016). These time periods enabled us to analyze the performance of the Financial Crisis on the U.S Agricultural banking sector as a whole. We applied a DEA-SVM model while accounting for the time dependency with the purpose of classifying the banks into four categories: (i) highly efficient (HE), (ii) highly inefficient (HI), (iii) efficient (E), and (iv) inefficient (I). Overall, the results revealed that technological progression declined due to financial crisis. More precisely, the performance of SVM declined during the financial crisis. The results show that the overall efficiency and performance using the integrated DEA-SVM during 2005-2006 and 2010-2016 were high. Furthermore, the integrated DEA-SVM had a lower performance during the financial crisis (2007-2009). The overall performance of all the kernels decreased during the financial crisis. Overall, the results show that the Agricultural banking sector is both efficient and stable over the time period being analyzed.

\section{Acknowledgement}

The authors would like to thank the anonymous referees for constructive comments on earlier version of this paper.

\section{References}

Andrews, D. F., \& Pregibon, D. (1978). Finding the outliers that matter. Journal of the Royal Statistical Society. Series B (Methodological), 40, 85-93.

Ataullah, A., \& Le, H. (2006). Economic reforms and bank efficiency in developing countries: the case of the Indian banking industry. Applied Financial Economics, 16(9), 653-663.

Azadeh, A., Ghaderi, S. F., Anvari, M., \& Saberi, M. (2007). Performance assessment of electric power generations using an adaptive neural network algorithm. Energy Policy, 35(6), 3155-3166.

Banker, R. D., \& Chang, H. (2006). The super-efficiency procedure for outlier identification, not for ranking efficient units. European Journal of Operational Research, 175(2), 1311-1320.

Banker, R. D., Gadh, V. M., \& Gorr, W. L. (1993). A Monte Carlo comparison of two production frontier estimation methods: corrected ordinary least squares and data envelopment analysis. European Journal of Operational Research, 67(3), 332-343.

Banker, R. D., Charnes, A., \& Cooper, W. W. (1984). Some models for estimating technical and scale inefficiencies in data envelopment analysis. Management science, 30(9), 1078-1092. 
Boyacioglu, M. A., Kara, Y., \& Baykan, Ö. K. (2009). Predicting bank financial failures using neural networks, support vector machines and multivariate statistical methods: A comparative analysis in the sample of savings deposit insurance fund (SDIF) transferred banks in Turkey. Expert Systems with Applications, 36(2), 3355-3366.

Cao, L. J., \& Tay, F. E. H. (2003). Support vector machine with adaptive parameters in financial time series forecasting. IEEE Transactions on Neural Networks, 14(6), 1506-1518.

Casu, B., \& Molyneux, P. (2003). A comparative study of efficiency in European banking. Applied Economics, 35(17), 1865-1876.

Charnes, A., Cooper, W. W., \& Rhodes, E. (1978). Measuring the efficiency of decision making units. European Journal of Operational Research, 2(6), 429-444.

Drake, L. (2001). Efficiency and productivity change in UK banking. Applied Financial Economics, 11(5), 557-571.

Favero, C. A., \& Papi, L. (1995). Technical efficiency and scale efficiency in the Italian banking sector: a non-parametric approach. Applied economics, 27(4), 385-395.

Fried, H. O., Lovell, C. K., Schmidt, S. S., \& Yaisawarng, S. (2002). Accounting for environmental effects and statistical noise in data envelopment analysis. Journal of productivity Analysis, 17(1-2), 157-174.

Hao, J., Hunter, W. C., \& Yang, W. K. (2001). Deregulation and efficiency: the case of private Korean banks. Journal of Economics and Business, 53(2-3), 237-254.

Holland, D. S., \& Lee, S. T. (2002). Impacts of random noise and specification on estimates of capacity derived from data envelopment analysis. European Journal of Operational Research, 137(1), 1021.

Kwiatkowski, D., Phillips, P. C., Schmidt, P., \& Shin, Y. (1992). Testing the null hypothesis of stationarity against the alternative of a unit root: How sure are we that economic time series have a unit root?. Journal of econometrics, 54(1-3), 159-178.

Martić, M., \& Savić, G. (2001). An application of DEA for comparative analysis and ranking of regions in Serbia with regards to social-economic development. European Journal of Operational Research, 132(2), 343-356.

Ondrich, J., \& Ruggiero, J. (2002). Outlier detection in data envelopment analysis: an analysis of jackknifing. Journal of the Operational Research Society, 53(3), 342-346.

Said, S. E., \& Dickey, D. A. (1984). Testing for unit roots in autoregressive-moving average models of unknown order. Biometrika, 71(3), 599-607.

Sealey Jr, C. W., \& Lindley, J. T. (1977). Inputs, outputs, and a theory of production and cost at depository financial institutions. The journal of finance, 32(4), 1251-1266.

Simar, L., \& Zelenyuk, V. (2011). Stochastic FDH/DEA estimators for frontier analysis. Journal of Productivity Analysis, 36(1), 1-20.

Tibshirani, R., Walther, G., \& Hastie, T. (2001). Estimating the number of clusters in a data set via the gap statistic. Journal of the Royal Statistical Society: Series B (Statistical Methodology), 63(2), 411423.

Racine, J. (2000). Consistent cross-validatory model-selection for dependent data: hv-block crossvalidation. Journal of Econometrics, 99(1), 39-61.

Vapnik, V. (2006). Estimation of dependences based on empirical data. Springer Science \& Business Media.

Vapnik, V. (1995). The Nature of Statistical Learning Theory. New York: Springer, 1995.

Vapnik, V. (1998). Statistical Learning Theory. Wiley, New York.

Wu, D. D., Yang, Z., \& Liang, L. (2006). Using DEA-neural network approach to evaluate branch efficiency of a large Canadian bank. Expert systems with applications, 31(1), 108-115.

Xu, Y., Zomer, S., \& Brereton, R. G. (2006). Support vector machines: a recent method for classification in chemometrics. Critical Reviews in Analytical Chemistry, 36(3-4), 177-188. 
(C) 2019 by the authors; licensee Growing Science, Canada. This is an open access article distributed under the terms and conditions of the Creative Commons Attribution (CC-BY) license (http://creativecommons.org/licenses/by/4.0/). 